\title{
Image Sand Style Painting Algorithm
}

\author{
Fan Hui ${ }^{1}$, Chen Zheng ${ }^{2}$ and Li Jinjiang ${ }^{1, *}$ \\ ${ }^{1}$ Key Laboratory of Intelligent Information Processing in Universities of Shandong (Shandong Institute of Business and Technology), \\ Yantai, China \\ ${ }^{2}$ Shandong Normal University, Jinan, China
}

Received: 9 Apr. 2013, Revised: 10 Aug. 2013, Accepted: 12 Aug. 2013

Published online: 1 Mar. 2014

\begin{abstract}
With the development of computer technology and the improvement of people's living level, the use of computers to deal with the photos is more and more popular. How to combine the computer and image perfectly will directly affect the quality of our image. Research of this article is how to manage the image by computer to desertification, that processing the image into desertification style. The result of traditional desertification method is not ideal, this article based on the matrix low-rank decomposition to processing image that decompose the matrix corresponding from the image, deal with the high order matrix into low order matrix and then reduce reduction into high order matrix, which simplifies the processing step and obtained good results.
\end{abstract}

Keywords: sand style, low-rank decomposition, level set interpolation

\section{Introduction}

Nowadays, ordinary image cant satisfy with the needs of the human, so different styles of image became more and more popular. The so-called styled images is by means of computer to simulate the artist's painting effect, so as to realize the rapid generation of different art style. The styled images have a few kinds as follows, such as the sketch, watercolor, oil painting, cartoon, sand painting, and so on, the main work is to transform the input image into the format that you need. For example, the oil painting style is that you input an original image, and the output is an oil painting. What discussed in our paper is one kind of stylized image, the sand painting style image. When you input an image, the output is a sand styled image. Draw the different styles of image belongs to a kind of non-realistic rendering. The purpose of the realistic rendering is to keep the nature information of the image and form the artistic style to what we need. Since the 1990s, nonrealistic rendering is taken more and more seriously in computer graphics, and its results of research are applied to all aspects of our society, such as in the field of computer animation, non-realistic drawing can make static images and dynamic image become visualize, "the moving qingming scroll" is one of it. Because each image corresponds to a matrix, that the image is composed of number and the computer is the processing of digital. So with the development of the computer technology, combine the image research with computer turns into one of the focuses of people research in image. Converting images into digital signal that work with computer is start in the 1920s. Now, the digital image processing is widely applied to various aspects of peoples life, such as justice, communications, medical, military, agriculture and industry, and so on. With the improvement of people's living standard, the peoples requirements of different style of image are more and more large. How to find a more simple way to achieve peoples demands become more and more important.

Due to the styled image can give people a better visual experience and it can be applied to many fields, such as scientific research, aerospace, military, industrial production, agricultural production, public security judicial, and so on. In People's Daily life, image processing plays a more and more important role, so the research on it will have more realistic significance.

\section{Related works}

With the rapid development of computer technology nowadays, the research of computer graphics is becoming more and more deeply at home and abroad. In domestic, the main researchers include state key laboratory of

\footnotetext{
*Corresponding author e-mail: lijinjiang @ gmail.com
} 
CAD\&\&G at Zhejiang University [1], [2] and national Chung Cheng University in Taiwan, and scholars from the Microsoft research in Asia. Their research areas include image rendering, sketch the simulation and the human face image and cartoons. Research results mainly include "magic brush" that developed by the human-computer interaction of CAS. Overseas, Haeberli proposed a very typical interactive drawing algorithm [3]. Research results mainly include Piranesi system that developed by Martin center architecture CAD laboratory at Cambridge university and electronic map technology center of Middlesex. Studies of stylized image are paid more and more attention in recent years, the study of images become more deeply, such as the research of human face. There is a way of training algorithm that is used to simulate the painters painting style. It locates facial features automatically when you input a new image and learns some drawing met hods of the geometric details of the outline of a face. Then it found the sample that matched the input image from the training library and optimize the combination, finally you will get the image you want. The traditional image processing mainly includes the following steps:

(1) Image digitization. To converted image into binary number that the computer can identify, we can use the computer to process images.

(2) Image sharpening processing. To enhance the area in the image that we interested in and weaken the area that we dont need.

(3) Image segmentation. To segmentation and divide the image into different classes according to the different grays level or any other feature of image.

(4) The reconstruction of the image. To form more realistic image according to the internal data of object. Because the image that we got has some missing area, it requires us to repair it. We can restore the missing area of the original image by the traditional image restoration algorithm. The traditional image restoration algorithm mainly includes the following several methods:

(1) Fuzzy enhancement. To enhancing the part of the image that we need and weaken the part that we dont need, then we will achieve the purpose of enhance the image quality.

(2) Level set difference reconstruction. The main process is to find an interpolation function to replace the original complex function method. To find the corresponding image level set, and then reconstruct the original image.

(3) Texture synthesis. The essence of it is to find a pixel on the edge of the missing area, then centered on the pixels to delimit an area, then go to the known area for the matched area, finally replaced the missing area by what you found.

(4) Based on partial differential equation restoration method. This is a professional and manual simulation method for recover image, this method is aimed to repair defect area through the iteration.
(5) Repair method based on image geometric model. This method is suitable for the image with a smaller missing area.

This paper is based on the traditional image restoration algorithm and introduced the matrix in low ranks algorithm into image restoration technology. The low-rank matrix algorithm is that transformed high order matrix into low rank matrix that corresponding the image. After processing, you should inverse transform the matrix into the original image. The algorithm is based on that the image and the matrix is one-to-one. Our image is stored by matrix in the computer. The low-rank decomposition matrix introduced in this paper not only makes the image repairing easier but also makes it closer to the real result.

\section{Sand style painting algorithm}

\subsection{Fuzzy enhancement}

Images are a mirror of real things, due to some reasons such as light and noise, it will inevitably produce some error, and the image fuzzy enhancement technology is an important technique to improve the image quality. It can improve image fuzzy condition, purposefully stressed or highlight some local characteristics of the image and weaken some information that we don't need. Thus we will get the image effect we want. We will introduce the classic image fuzzy enhancement algorithm-Pal and King fuzzy enhancement algorithm [4] [5] [6] as follows. Set the corresponding two-dimensional matrix with the image which the gray is $\mathrm{X}$ and the order number is $M \times N$ as follow:

$$
X=\left(\begin{array}{cccc}
\frac{p_{11}}{X_{11}} & \frac{p_{12}}{X_{12}} & \ldots & \frac{p_{1 N}}{X_{1 N}} \\
\frac{p_{21}}{X_{21}} & \frac{p_{22}}{X_{22}} & \ldots & \frac{p_{2 N}}{X_{2 N}} \\
\cdots & \ldots & \ldots & \ldots \\
\frac{p_{M 1}}{X_{M 1}} & \frac{p_{M 2}}{X_{M 2}} & \cdots & \frac{p_{M N}}{X_{M N}}
\end{array}\right)
$$

Among it $\frac{p_{i j}}{X_{i j}}$ the membership that the coordinates of pixels at $(x, y)$ of the image corresponding to a particular gray scale $X_{i j}$ is $P_{i j}\left(0 \leq P_{i j} \leq 1\right)$. Membership function has a great influence on the choice of detection in the images. The membership function of $\mathrm{P}$ is:

$$
P_{i j}=F\left(x_{i j}\right)=\left[1+\frac{(L-1)-x_{i j}}{F_{d}}\right]^{-F_{e}}
$$

Among it, $F_{d}$ and $F_{e}$ called reciprocal fuzzy factors and index fuzzy factors. Under normal circumstances $F_{\varepsilon}=2$ . To process the image with fuzzy enhancement, we first should use the changing:

$$
\left\{\begin{array}{l}
\mu_{i j}=I\left(\mu_{i j}\right)=I_{1}\left(I_{r-1}\left(\mu_{i j}\right)\right), r=1,2 \ldots \\
I_{1}\left(\mu_{i j}\right)=\left\{\begin{array}{l}
2 \mu_{i j}^{2} \quad 0 \leq \mu_{i j} \leq 0.5 \\
1-2\left(1-\mu_{i j}\right)^{2} 0.5<\mu_{i j} \leq 1
\end{array}\right.
\end{array}\right.
$$

Among it, the effect of formula (1) is to magnify (when $\mu_{i j}>0.5$ ) or decrease (when $\mu_{i j}<0.5$ ) the value 
of $\mu_{i j}$. Inverse transformation of $\mu_{i j}$, we will get the image $X^{\prime}$ after fuzzy enhancement of the original image. The gray value $x_{i j}$ of the pixel at the coordinates of $(\mathrm{x}, \mathrm{y})$ is $x_{i j}=T^{-1}\left(\mu_{i j}\right)$. We know that dealing with the blurred image is that mapping the pixel of the image from the spatial domain to fuzzy field and use a fuzzy enhancement operator to iterative enhancement arithmetic in fuzzy domain and finally using the inverse function of fuzzy membership function to inverse mapping the pixel back to space domain. In our study of grade 256 in the image, the maximum gray value of the image is 256 , far less than the number of the pixels of the image. So, use the gray level to fuzzy enhance the image is easier than use the image pixels to fuzzy enhance the image. Based on the gray scale and use MATLAB software to achieve the specific steps are as follows:

(1) Mapping the gray level $[0,255]$ from spatial domain to fuzzy field

(2) Have $r$ times enhancement arithmetic based on fuzzy operator in fuzzy domain

(3) Inverse transforms the data got by fuzzy enhancement to spatial domain

(4) Converse the gray values of the $M \times N$ pixels into fuzzy enhancement, at this moment we only need to traverse each pixel of the image at a time and do simple assignment operation

There are some shortcomings in Pal fuzzy enhancement algorithm. Firstly, the value at the turning point in the fuzzy enhancement function is 0.5. For different images, obviously threshold is not necessarily the same, so the value will not necessarily suitable for every image. Secondly, the algorithm adopts the form of fuzzy set membership function of power function transform the normal space into generalized fuzzy space sets. The computation is big and will loss part of the gray value of the boundary in image information. Here is an improved image enhancement algorithm [7] . First of all, we should eliminate the influence of threshold and define a new membership function:

$$
p_{i j}=F\left(x_{i j}\right)=\left\{\begin{array}{l}
s_{1} \operatorname{tg}^{2}\left(\frac{\pi x_{i j}}{4(L-1)}\right) \quad 0 \leq x_{i j} \leq x_{T} \\
1-s_{2}\left(1-\operatorname{tg} \frac{\pi x_{i j}}{4(L-1)}\right)^{2} \quad x_{T}<x_{i j} \leq L-1
\end{array}\right.
$$

Among it

$$
s_{1}=\frac{x_{T}}{(L-1) \operatorname{tg}^{2}\left(\frac{\pi x_{T}}{4(L-1)}\right)} s_{2}=\frac{L-1-x_{T}}{(L-1)\left(1-\operatorname{tg} \frac{\pi x_{T}}{4(L-1)}\right)^{2}}
$$

\subsection{Level set interpolation}

The interpolation problem means we've got a function $y=f(x)$ and we know the values $f\left(x_{0}\right), f\left(x_{1}\right), \cdots, f\left(x_{n}\right)$ in the corresponding nodes $x_{0}, x_{1}, \ldots, x_{n}$. Then we construct a function $\phi(x)$ and make it content that $y=f\left(x_{i}\right)=\phi\left(x_{i}\right)$ [8] in the corresponding nodes $x_{0}, x_{1}, \ldots, x_{n}$. We call $\phi(x)$ interpolation function. Almahs put forward the AMLE model and proved the following second order partial differential equations have the only viscous solution:

$$
\left\{\begin{array}{l}
\nabla^{2} u\left(\frac{\nabla u}{|\nabla u|}, \frac{\nabla u}{\nabla u}\right)=0 \text { in } \Omega \\
\left.u\right|_{\partial \Omega}=\phi_{1},\left.u\right|_{c_{1}}=\phi_{c_{i}} i=1,2, \ldots, m \\
u\left(x_{i}, y_{i}\right)=u_{i} i=1,2, \ldots, N
\end{array}\right.
$$

Among it, $\partial \Omega$ is the boundary of $\Omega, C_{i}$ is a curve of $\Omega,\left(x_{i}, y_{i}\right)$ is a node in $\Omega$.

Due to the steady state solution of elliptic partial differential equations are consistent with the steady state solution of the corresponding parabolic partial differential equations, so the level set interpolation of the image can be disposed by the parabolic partial differential equation that corresponding with equation (2). The last two expressions of (2) are the general boundary conditions. We can get by (2):

$$
\nabla^{2} u\left(\frac{\nabla u}{|\nabla u|}, \frac{\nabla u}{|\nabla u|}\right)=\frac{u_{x}^{2} u_{x x}+2 u_{x} u_{y}+u_{y}^{2} u_{y y}}{u_{x}^{2}+u_{y}^{y}}=\frac{\partial^{2} u}{\partial \eta^{2}}
$$

Using central difference explicit difference scheme $u_{i j}^{n+1}=u_{i j}^{n}+\Delta t N\left(u^{n}\right)_{i j}$ can get the solution.

For a gray scale image $u(x, y)$, the level set of the image is defined as:

$$
x_{\lambda}(u)=\{(x, y) \in \Omega ; u(x, y) \geq \lambda\}, \lambda \in\left(u_{\min }, u_{\max }\right)
$$

Among it, $\lambda$ is the value $u_{\min }, u_{\max }$ is the minimum value and the maximum value. The level set of the image said here is different with the level set of the embedded function. It has a certain area, while the latter is the border of $x_{\lambda}$. Set $x_{\lambda}$ is a collection of two-dimensional, it contains multiple separate connected components and each connected components is zero area. According to this definition, every image takes different values of $\lambda$, then we can get the corresponding image level set. As we known all the level set of an image, then we can reconstruct the original image completely by the level set:

$$
u(x, y)=\sup \left\{\lambda,(x, y) \in\left\{\chi_{\lambda}(u)\right\}\right\}
$$

So, we usually referred formula (4) as the level set decomposition formula of the image, formula (5) is called the level set reconstruction formula.

\subsection{Patchmatch}

Patchmatch is a kind of fast algorithm that computing the fuzzy correspondence between adjacent area of the two patches. The algorithm has been applied to many fields of image and video processing now. These areas include texture synthesis, image and video restoration, image stitching and so on. Connelly Barnes and Eli Shechtman proposed an approximate nearest neighbor algorithm [9] 
and the emphasis of the algorithm is how to calculate the relevant patch. First of all, we define a nearest neighbor function (NNF) $f: A \mapsto R^{2}$ for the distance function between two patches. Define all possible patches of the image $\mathrm{A}$ (the center of the patch). Consider coordinates a of the patch in image A and it's adjacent area $b$ in image B. $f(a)$ only means $b-a$. We refer to the value of $f$ as compensation and they are stored in a matrix which the dimension is same as matrix A. Then, the following gives the main steps of the algorithm:

(1) Initialization

The adjacent area can be initialized by distribute the random values to the region or use the apriori information. When we initialize the random offset, we use the independent sample to traverse the entire range of the image B. But if we only use the initial guess, the algorithm may lead to a poor local minimum. When we retain the quality of this prior, we still need to preserve the ability to escape from such minimum. We use the random initial value to perform the previous iteration algorithm, then merged with the sampling initial value at the smaller parts of $\mathrm{D}$ and complete the rest of the iteration.

(2) Iteration

After initialization, we perform an iterative process that improves the NNF. The algorithm of each iteration process is as follows: check the coordinate scanning sequence(from left to right, from top to bottom) and each have to undergo communication after random search. These operations are interleaved at the patch level: if $P_{j}$ and $S_{j}$ means separate spread and the random search of patch $j$, then we can go on with the following order: $P_{1}, S_{1}, P_{2}, S_{2}, \ldots, P_{n}, S_{n}$. We can go on with the following three steps:

(i)Spread

We try to use the known coordinates $f(x-1, y)$ and $f(x, y-1)$ to improve the function $f(x, y)$ and assume that this patch coordinates is likely the same. For example, if it has a good map at $(\mathrm{x}-1, \mathrm{y})$, we will try to use this translation mapping a pixel to the right side of us. Using $D(v)$ as the distance between patch at the coordinates $(x, y)$ of $\mathrm{A}$ and the patch $(x, y)+v$ of $\mathrm{B}$. We give a new value as the independent variable:

$$
\{D(f(x, y)), D(f(x-1, y)), D(f(x, y-1))\}
$$

The effect is that if have a correct map in a coherent region $\mathrm{R}$, then all the region $\mathrm{R}$ and the right of $(x, y)$ will filled with correct mapping. In addition, we check up reverse scanning coordinate to pass information up or to the left through iteration and use $f(x+1, y)$ and $f(x, y+1)$ as substitute for our coordinates.

(ii)Random search

Let $v_{0}=f(x, y)$. We attempt to improve $f(x, y)$ by testing a sequence of candidate offsets at an exponentially decreasing distance from $v_{0}$

$$
u_{i}=v_{0}+\omega \alpha^{i} R_{i}
$$

Among it, $R_{i}$ is a uniform random number of $[-1,1] \times$ $[-1,1] . \omega$ is a large maximum search "radius" and $\alpha$ is a fixed ratio between the size of the search window. We detect the patch, let $i=0,1,2, \ldots$ until the current search radius $\omega \alpha^{i}$ is less than one pixel. In our application, $\omega$ is the biggest image size and $\alpha=1 / 2$ except the special instructions. Note that the search window must be clamped to the boundary B.

(3) The stop standard

Although, different applications may have different end conditions, we found the number of iterations when it works well. All the results here shows are need four to five times of iterative computation altogether and it is always convergence after NNF.

\subsection{Brightness adjustment}

Each image will be darker part. There will be a bright part and we call the brightest part of high optical modulation, dark as part of a low optical modulation and the remaining part is called the midtones. Image brightness adjustment means to adjust the brightness of the image, making the image better visual effect and highlighting the part of the image we need to improve the sharpness of the image. Image brightness adjusting method generally has the following two kinds: one is directly in the original image is adjusted, another is the image mapping. The image mapping is to map the image to another area that we called the transform domain and then transform image in the domain. Finally, we can inverse transform the result to get the result we want. There are several specific processing methods as follows:

(1) the image gray-scale transformation, that is to expand the dynamic range of the image, the image feature becomes apparent;

(2) image smoothing, which removes noise and false contour in the image;

(3) image sharpening, the purpose of image sharpening is to make the part of the image we need becomes more prominent and enhance the part that is useful to us;

(4) homomorphism norm of images, namely the method that compress the brightness component of the image in the frequency domain and enhance image contrast at the same time;

In order to adjust the brightness of image, the first gray-scale image (color image is directly converted into a gray scale image) corresponding histogram is divided into $080,81130,131180,181255$ of these four sections, and the proportion of these four sections are denoted by $P E R_{1}, P E R_{2}, P E R_{3}, P E R_{4}$ [10].

Among it, num $_{p_{i}}$ represents the number of pixel values of pixels of $i$, num $_{p}$ is the total number of image pixels.

Then, change the corresponding pixel gray value of the image through the traditional histogram piecewise linear transform method and thereby you can adjust the color of 
image.

$$
\left\{\begin{aligned}
\text { PER }_{1} & =\sum_{i=0}^{80} \text { num }_{p_{i}} / \text { num }_{p} \\
\text { PER }_{2} & =\sum_{i=81}^{130} \text { num }_{p_{i}} / \text { num }_{p} \\
\text { PER }_{3} & =\sum_{i=131}^{180} \text { num }_{p_{i}} / \text { num }_{p} \\
\text { PER }_{4} & =\sum_{i=181}^{255} \text { num }_{p_{i}} / \text { num }_{p}
\end{aligned}\right.
$$

\subsection{Texture Synthesis}

Image restoration based on texture synthesis technique is a study of computer vision, computer graphics, and it is an important hotspot. It can not only repair the lack or distortion area of the smaller images, but also can repair the damaged part details. Principle of texture image synthesis technology is based on the known region to repair the unknown regions. Namely, select a pixel on the edge of the loss or distort zone, and then match the small approximate areas that close to the pixel and its neighbors in the known area according to the neighboring pixels. Criminisi presents a texture synthesis image completion based on block [11], which consists of three steps:

(1) calculate the optimum weights, select a region with the highest priority to fill;

(2) select an area which is closest to the area needed to fill in the known area; fill.

(3) put the area that we find into the area that need to

The detailed steps are as follows [12] [13]:

(1) Calculate the optimum weights Firstly we need to calculate the most weight image block, the center for image block weight record for $p$ :

$$
P(p)=C(p) * D(p)
$$

Among it, $C(p)$ is called the trust factor represents the $p$ point reliability of information; $D(p)$ called data factor represents the projection of $\partial \Omega$ light at the boundary of $C(p)=\frac{\sum_{q \in \psi_{p} \cap(I-\Omega)} C(q)}{\left|\psi_{p}\right|}, D(p)=\frac{\left|\nabla I_{p}^{\perp} \bullet \vec{n}_{p}\right|}{\alpha} . I$ represents the entire image, $\Omega$ represents area of the image that need to be repaired, $\psi_{p}$ represents an image block that need to repair, $\left|\psi_{p}\right|$ represents the area of the image block, $\alpha$ is the normalization factor (gray image is 255), $n_{p}$ represents the unit of the vertical vector of $p$ at the point on the boundary $\partial \Omega$.

(2) Select the optimal region In the known region $(I-$ $\Omega)$ according to the formula $\underset{\hat{q}}{\psi_{\hat{A}}}=\arg \min _{\psi_{q} \in(I-\Omega)} d\left(\psi_{p}, \psi_{q}\right)$ to obtain the optimal similarity block in the area that need to fill. Where $d\left(\psi_{\hat{p}}, \psi_{q}\right)$ is the distance between $\psi_{p}$ and $\psi_{q}, \psi_{\hat{p}}$ is means the block that has the minimum distance with $\psi_{p}$.
(3) Fill the missing area with the optimal area Feng proposed an improved algorithm based on Criminisi's algorithm [14]. The algorithm reduces the search for matching block range and gives a choice of optimal block criterion in the known region, and improves the efficiency of our codes for the optimal block. The algorithm that presented by H. Yamauchi [15] al et. , combined the traditional algorithms with the multi-resolution texture synthesis algorithm to repair the image with widespread damage under the condition of multi-resolution.

\section{Experimental Results}

Figure 1 is the real sand painting image. Figure 2 is the original images and the corresponding experimental results. We can see clearly that, in this paper, the result of the sand painting is very close to the real effect of sand painting. Figure 2 shows the results of sand paintings that under different shade. We can see that the contrast obvious places of the images are also obvious in the result of the sand paintings. It can be seen that the method in this paper has a good sand painting effect for images that under different intensity.

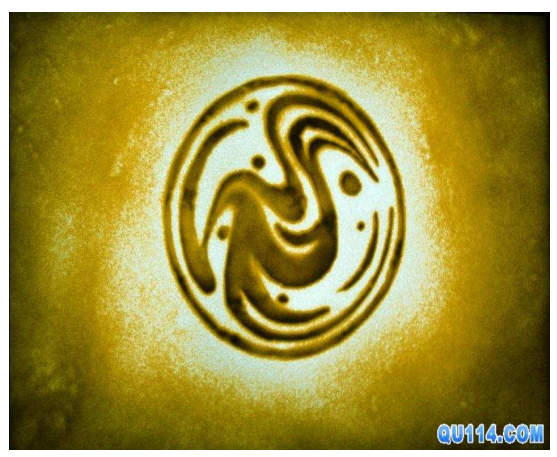

Fig. 1: Real sand painting image

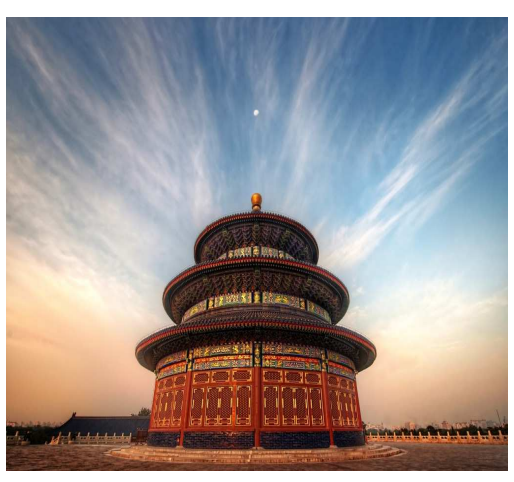

(a) 


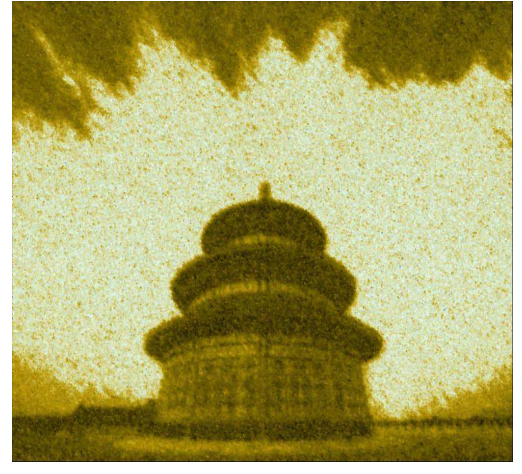

(b)

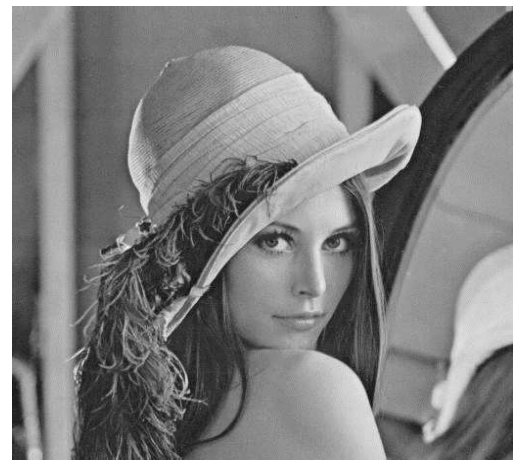

(c)

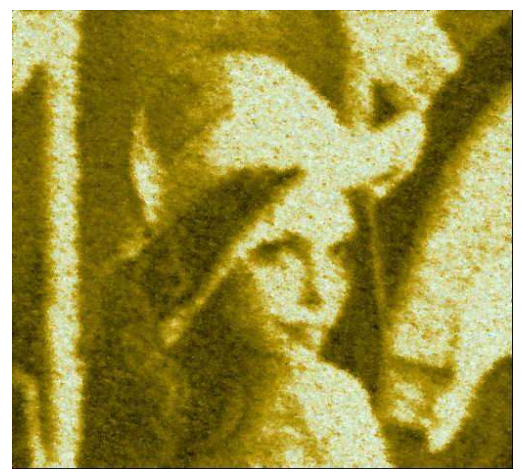

(d)

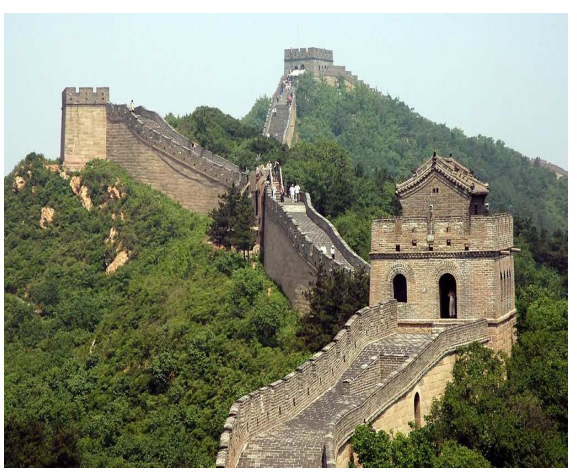

(e)

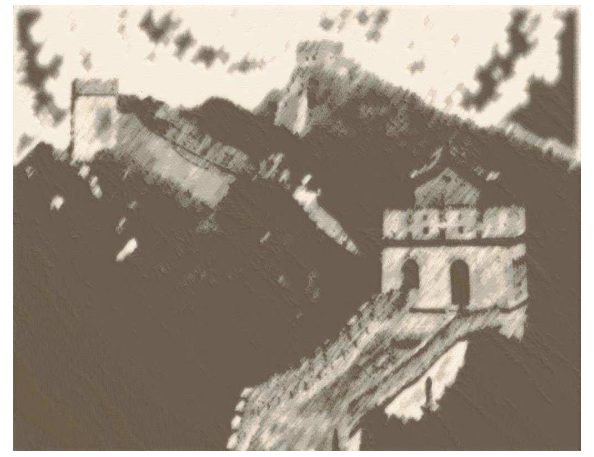

(f)

Fig. 2: Image Sand Style Painting

We can see from the result of the experiment, the result can express the texture of the image clearly. The contrast of images is obvious, and the sense of level is stronger. Such as the outline of the building in figure 2(a) and the outline of the people in figure 2(c). And in the figure 2(e), we can see very clear that sand painting effect is also very good at the place where the intensity jumping bigger in the image. Thus we can get that, it has better effect of sand painting in shade contrasts place in this paper.

On the processing speed, this paper also has obvious advantages. Because of that this article cites a matrix decomposition algorithm for image preprocessing. It makes the order of the matrix that corresponding to the image reduced, so as to make the program running time complexity reduced. The traditional method that processing the matrix which corresponding to the original image, not only will make the dealing process more complex, but also the result may be not very ideal. But the method of this paper is to reduce the program's running time complexity but also get better treatment effect. Of course, the effect of the sand painting in this paper also has subtle gap compared with the real sand images(include hand-painted artist and real desertification scenery). And manually mapped by the sand painting can reflect the author's inner feelings, these are realistic drawing cannot reach.

\section{Conclusion}

Sand style painting image belongs to a kind of styled image and it satisfies the demand for this kind of image style. This image and other stylized images give people a different visual experience. This paper introduces an image processing algorithms in the basis of former research, namely the low-rank matrix decomposition. Combining it with image processing, we can get better effect which is mainly composed of the following: (1) Simplify the processing steps. Low-rank matrix decomposition is to decompose the matrix corresponding 
to the image into a lower order matrix which processing up more simple and convenient. (2) We can get a good result throw the low-rank matrix decomposition according to the experimental.

\section{Acknowledgement}

This work was supported by the National Natural Science Foundation of China (NSFC) under Grant (61173173, 61272430); Shandong province Natural Science Foundation (ZR2011FM035). Yantai science and technology development plan (2011055).

\section{References}

[1] Zhang Junsong Yu Jinhui, Mao Guogong Ye Xiuzi. Using autoregressive (AR) and stratifiedsampling to generate cursive stroke texture. Journal of computer-aided design \& computer graphics, 12, 1439-1403 (2007).

[2] Yu Jinhui Xu Xiaogang, Peng Qunsheng. Computer generated animation cartoon smoke. Chinese Journal of computers, 23, 987-990 (2000).

[3] Hausner A. Simulating decorative mosaic. Proc. Siggraph, 2001, 573-578 (2001).

[4] S K Pal, R A King. Image Enhancement Using Fuzzy Sets. Electronics Letters, 16, 376-378 (1980).

[5] J Han, K K Ma. Fuzzy color histogram and its use in color image retrieval. IEEE Trans. on Image Process, 11, 944-52 (2002).

[6] S K PaI, R A King. On Edge Detection of X-Ray Images Using Fuzzy Sets. IEEE Trans Pattern Anal Mach Intell, 5, 69-77 (1983).

[7] Liu XiWen, Jiang Yanrong, Luo Xianguang. An improved image fuzzy enhancement algorithm. Computer Engineering and Applications, 44, 50-51 (2008).

[8] Zhang Jie. Image reconstruction of B spline amplification algorithm based on level set. Computer simulation, 29, 269272 (2012).

[9] Connelly Barnes, Eli Shechtman. PatchMatch: A Randomized Correspondence Algorithm For Structural Image Editing. ACM SIGGRAPH 2009, Article No. 24, (2009).

[10] Xu Lei. Based on the classification and improve the dichotomy of license plate character segmentation and. Shandong science, 25, 2-3 (2012).

[11] CriminisiA, Perez P, Toyama K. Region filling and object removal by exemplar-based image inpainting. IEEE Transactions on Image Processing, 13, 1200-1212 (2004).

[12] A. Criminisi, P. Perez and K. Toyama. Region Filling and Object Removal by Exemplar Based Inpainting. IEEE Transactions on image processing, 13, 1200-1212 (2004).

[13] A. Criminisi, P. Perez, K. Toyama. Object Removal by Exemplar-Based Inpainting. Proceedings of the 2003 IEEE Computer Society Conference on Computer Vision and Pattern Recognition(CVPR03), 721-728 (2003).

[14] Tang F, Ying Y T, Wang J, et al. A novel texture synthesis based algorithm for object removal in photographs. Proceedings of Ninth Asian Computing Science Conference, 248-258 (2004).
[15] H. Yamauchi, J. Haber and H. Seidel. Image Restoration Using Multiresolution Texture Synthesis and Image Inpinting. Proceedings Computer Graphics International, 120-125 (2003).

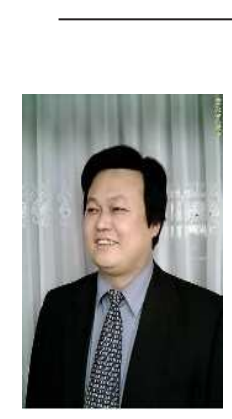

Hui Fan was born in 1962, Ph.D., professor, master tutor, the $\mathrm{CCF}$ senior member, committee member of virtual reality and visualization technology. The research direction of computer graphics, image processing, virtual reality.

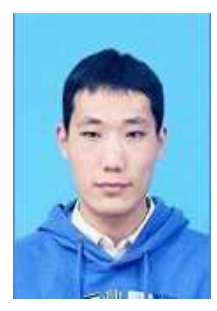

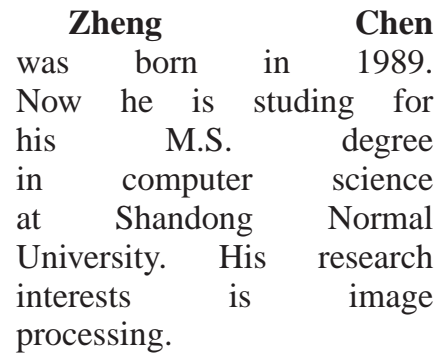

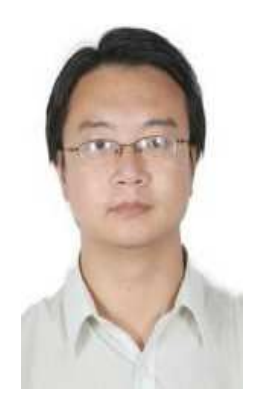

Jinjiang Li was born in 1978, associate professor and $\mathrm{Ph} . \mathrm{D}$. $\mathrm{He}$ received the master's degree in applied computer from TaiYuan University of technology in 2004. From 2004 to 2006, he was a assistant engineer at institute of computer science \& technology of Peking University. He awarded a Ph.D. degree in computer from Shandong University in 2010, and now work at Shandong Institute of Economic \& Techonlogy. His research interests include computer graphics, computer-aided geometry design and digital image processing. 